\title{
Photometric Invariant Projective Registration Using ECC Maximization
}

\author{
Georgios D. Evangelidis and Emmanouil Z. Psarakis \\ Department of Computer Engineering and Informatics \\ University of Patras, 26500 Rio-Patras, Greece \\ email:\{evagelid, psarakis\}@ ceid.upatras.gr *
}

\begin{abstract}
The ability of an algorithm to accurately estimate the parameters of the geometric transformation which aligns two image profiles even in the presence of photometric distortions can be considered as a basic requirement in many computer vision applications. Projective transformations constitute a general class which includes as special cases the affine, as well as the metric subclasses of transformations. In this paper the applicability of a recently proposed iterative algorithm, which uses the Enhanced Correlation Coefficient as a performance criterion, in the projective image registration problem is investigated. The main theoretical results concerning the iterative algorithm and an efficient approximation that leads to an optimal closed form solution (per iteration) are presented. Furthermore, the performance of the iterative algorithm in the presence of nonlinear photometric distortions is compared against the leading Lucas-Kanade algorithm by performing numerous simulations. In all cases the proposed algorithm outperforms the Lucas-Kanade algorithm in convergence speed and robustness against photometric distortions under ideal and noisy conditions.
\end{abstract}

\section{Introduction}

Alignment or registration problem consists in finding a transformation which aligns two image profiles. Such correspondence problems arise often in practice with the most common cases being motion and optical flow estimation [1, 2, 4, 6, 8, 9] tracking [7] and stereo correspondence problem $[10,11]$ to name a few. The first critical step towards its solution is the choice of the appropriate class of geometric transformations (parametric models [13]) that can model this mapping. This choice is strictly related with

*This work was supported by the General Secretariat for Research and Technology of Greek Government as part of the project "XROMA", PENED 01. the application at hands and may be restricted from the strategy that is followed to solve the alignment problem [3]. The class of projective transformations and in particular several special cases as affine, similariry transformations and pure translation have been in the center of attention in many applications $[3,6,7]$.

Once the parametric transformation has been defined the alignment problem reduces into a parameter estimation problem. Therefore, the second critical step in solving the alignment problem is the definition of an appropriate objective function and the solution of the induced optimization problem. Most of the used measures are $l_{p}$ based norms of the error between the whole image profiles (pixel-based techniques) or between specific feature of image profiles (feature-based techniques) [13], with the $l_{2}$ norm being by far the most widely used [2, 3, 6, 7, 10, 12]. This $l_{2}$ based objective function is usually referred as SumSquared-Differences (SSD) measure. Some variations of this technique have also been proposed, especially for the optical flow problem, adding spatial smoothness constraints and/or regularization parameters [8]. Robustified versions of the above mentioned measures that reduce their sensitivity in the presence of outliers have also been proposed [4].

Independently of the used measure, the parameter estimation problem results in a non-linear optimization problem and the alignment techniques, depending on the strategy they follow to solve it, can be broadly classified into two categories. Namely, gradient-based or differential and direct search techniques. Gradient based techniques because of their low computational cost can be considered as more well fitted to CV applications. However, homogeneous areas, single slanted edges (aperture problem) [9] as well as large motions constitute some pathological cases where these techniques fail to give meaningful results. On the other hand, direct search techniques can easily manage large motions, since the range of the search area is in our disposal. The drawbacks of these techniques are their heavy complexity and the fact that the precision of the optimum solution is affected from the quantization step [6]. Efforts to reduce complexity by adopting interpolation instead of 
fine quantization or hybrid techniques that combine the two classes can be found in $[2,12]$.

A common assumption encountered in most of the existing techniques is the brightness constancy of corresponding points or regions in the two profiles [9]. However, this assumption is valid only in specific cases [9] and especially under varying illumination conditions it is violated. In a real world application it is vital an alignment algorithm to be capable in taking into account illumination changes. Several alignment techniques that compensate photometric distortions in contrast and brightness have been proposed in the literature $[6,9,10]$. Moreover, in [7] alignment techniques which make use of a set of basis images for handling arbitrary lighting conditions as well as more sophisticated spatially dependent photometric models [1] have also been proposed.

In [5] a modification of the correlation coefficient as a performance criterion for the image alignment problem is proposed. The proposed modification provides certain desirable invariance properties with respect to photometric distortions. Since the similarity measure is a highly nonlinear function of the warp parameters, they developed an iterative maximization technique. Based on an efficient approximation a closed form solution (per iteration) of the iterative algorithm is presented. Moreover, the authors used the class of affine transformations to model the warping process and their iterative scheme compared against the LucasKanade algorithm with the help of numerous simulations. In all cases the algorithm exhibited a noticeable performance improvement over the Lucas-Kanade scheme regarding convergence speed as well as in probability of convergence.

In this paper we present part of the theoretical results contained in [5], perform a number of simulations in order to evaluate the performance of the above mentioned scheme in the presence of nonlinear photometric distortions, and compare it against the well known Lucas-Kanade alignment algorithm. In all experiments we use the class of projective transformations to model the warping process.

The remainder of this paper is organized as follows. In Section 2, we briefly formulate the parametric image alignment problem. In Section 3, we present the $l_{2}$ based objective function and comment on its equivalency with the Enhanced Correlation Coefficient function as well as its relation to the two most popular approaches based on the SSD measure. In the same section the proposed gradient based iterative scheme and the basic non-linear optimization problem are defined. In addition, the optimal closed form solution of the basic optimization problem is given. In Section 4 , we apply the proposed technique in a number of experiments and perform detailed comparisons with the LucasKanade alignment algorithm. Finally, Section 5 contains our conclusions.

\section{Problem Formulation}

In this section we briefly introduce the problem of alignment of two image profiles. To this end, let us assume that a reference image $I_{r}(\mathbf{x})$ and a warped image $I_{w}\left(\mathbf{x}^{\prime}\right)$ are given, where $\mathbf{x}=[x, y]$ and $\mathbf{x}^{\prime}=\left[x^{\prime}, y^{\prime}\right]$ denote coordinates. Suppose also that we are given a set of coordinates $\mathcal{S}=\left\{\mathbf{x}_{i} \mid i=1, \ldots, K\right\}$ in the reference image, which is called target area. Then, the alignment problem consists in finding the corresponding coordinate set in the warped image.

By considering that a transformation model $T(\mathbf{x} ; \mathbf{p})$ where $\mathbf{p}=\left(p_{1}, p_{2}, \ldots, p_{N}\right)^{t}$ is a vector of unknown parameters is given, the alignment problem is reduced to the problem of estimating the parameter vector $\mathbf{p}$ such that

$$
I_{r}(\mathbf{x})=\Psi\left(I_{w}(T(\mathbf{x} ; \mathbf{p})) ; \boldsymbol{\alpha}\right), \mathbf{x} \in \mathcal{S}
$$

where transformation $\Psi(I, \boldsymbol{\alpha})$ which is parameterized by a vector $\boldsymbol{\alpha}$, accounts for possible photometric distortions that violate the brightness constancy assumption, a case which arises in real applications due to different viewing directions and/or different illumination conditions.

The goal of most existing algorithms is the minimization of the dissimilarity of the two image profiles, providing the optimum parameter values. Dissimilarity is usually expressed through an objective function $E(\mathbf{p}, \boldsymbol{\alpha})$ which involves the $l_{p}$ norm of the intensity residual of the image profiles. A typical minimization problem has the following form

$$
\min _{\mathbf{p}, \boldsymbol{\alpha}} E(\mathbf{p}, \boldsymbol{\alpha})=\min _{\mathbf{p}, \boldsymbol{\alpha}} \sum_{\mathbf{x} \in \mathcal{T}}\left|I_{r}(\mathbf{x})-\Psi\left(I_{w}(\boldsymbol{\phi}(\mathbf{x} ; \mathbf{p})), \boldsymbol{\alpha}\right)\right|^{p}
$$

Solving the above defined problem is not a simple task because of the nonlinearity involved in the correspondence part. Computational complexity and estimation quality of existing schemes depends on the specific $l_{p}$ norm and the models used for warping and photometric distortion. As far as the norm power $p$ is concerned most methods use $p=2$ (Euclidean norm). This will also be the case in the approach we briefly present in the next section.

\section{The Alignment Algorithm}

It is more convenient at this point to define the reference vector $\mathbf{i}_{r}$ and the warped vector $\mathbf{i}_{w}(\mathbf{p})$ as follows

$$
\mathbf{i}_{r}=\left[\begin{array}{c}
I_{r}\left(\mathbf{x}_{1}\right) \\
I_{r}\left(\mathbf{x}_{2}\right) \\
\vdots \\
I_{r}\left(\mathbf{x}_{K}\right)
\end{array}\right], \mathbf{i}_{w}(\mathbf{p})=\left[\begin{array}{c}
I_{w}\left(T\left(\mathbf{x}_{1} ; \mathbf{p}\right)\right) \\
I_{w}\left(T\left(\mathbf{x}_{2} ; \mathbf{p}\right)\right) \\
\vdots \\
I_{w}\left(T\left(\mathbf{x}_{K} ; \mathbf{p}\right)\right)
\end{array}\right]
$$


and denote the by $\overline{\mathbf{i}}_{r}$ and $\overline{\mathbf{i}}_{w}(\mathbf{p})$ the zero-mean versions of the reference and warped vector respectively. We then propose the following $l_{2}$ based criterion to quantify the performance of the warping transformation with parameters $\mathbf{p}$

$$
E_{E C C}(\mathbf{p})=\left\|\frac{\overline{\mathbf{i}}_{r}}{\left\|\overline{\mathbf{i}}_{r}\right\|}-\frac{\overline{\mathbf{i}}_{w}(\mathbf{p})}{\left\|\overline{\mathbf{i}}_{w}(\mathbf{p})\right\|}\right\|^{2},
$$

where $\|\cdot\|$ denotes the usual Euclidean norm.

It is clear from (4) that this criterion is invariant to possibly existing contrast and/or brightness changes since involved vectors are zero-mean and normalized. So, to a first approximation, we can concentrate on the geometric transformation putting aside the photometric one. These characteristics clearly support our choice to adopt this criterion for the image alignment problem.

By assuming that photometric distortion is limited only to global brightness and contrast changes, we can derive the proposed criterion in a different way. This will help us in related it to the two, currently most popular SSD approaches in the literature. Under the above mentioned assumption on photometric changes, and by defining the following performance measure

$$
E(\mathbf{p}, \boldsymbol{\alpha})=\left\|\alpha_{1} \mathbf{i}_{w}(\mathbf{p})+\alpha_{2}-\mathbf{i}_{r}\right\|^{2},
$$

where $\boldsymbol{\alpha}=\left[\alpha_{1} \alpha_{2}\right]^{t}$ is the parameter vector for the photometric transformation, the proposed measure can easily be proved that is equivalent with the following constrained minimization problem

$$
E_{E C C}(\mathbf{p})=\min _{\alpha_{1} \geq 0, \alpha_{2}} E(\mathbf{p}, \boldsymbol{\alpha}) .
$$

If we drop the constraint $\alpha_{1} \geq 0$ then the minimization of the objective function in (5) is the optimization problem proposed by Fuh and Maragos [6].

An alternative measure arises if in (5) we interchange the roles of $\mathbf{i}_{w}$ and $\mathbf{i}_{r}$, that is,

$$
E(\mathbf{p}, \boldsymbol{\alpha})=\left\|\alpha_{1} \mathbf{i}_{r}+\alpha_{2}-\mathbf{i}_{w}(\mathbf{p})\right\|^{2} .
$$

This is the approach adopted by Lucas-Kanade [10] and is known to generate the most widely used algorithm in practice.

\subsection{A Nonlinear Maximization Problem}

Let us now concentrate ourselves on the minimization of the proposed measure. Since the residual in (4) is based on zero-mean and normalized vectors, it is straightforward to prove that minimizing $E_{E C C}(\mathbf{p})$ is equivalent to maximizing the enhanced correlation coefficient [11]

$$
\rho(\mathbf{p})=\hat{\mathbf{i}}_{r}^{t} \frac{\overline{\mathbf{i}}_{w}(\mathbf{p})}{\left\|\overline{\mathbf{i}}_{w}(\mathbf{p})\right\|}
$$

where $\hat{\mathbf{i}}_{r}$ is the normalized reference vector. Notice that even if $\overline{\mathbf{i}}_{w}(\mathbf{p})$ depends linearly on the parameter vector $\mathbf{p}$, the resulting objective function is still nonlinear with respect to $\mathbf{p}$ due to the normalization of the warped vector. This of course suggests that its maximization requires nonlinear optimization techniques.

In order to maximize $\rho(\mathbf{p})$ we are going to use a gradient-based iterative approach. More specifically, we are going to replace the original optimization problem by a sequence of secondary optimizations. Each such optimization relies on the outcome of its predecessor thus generating a sequence of parameter estimates which hopefully converges to the desired optimizing vector of the original problem. Notice that, at each iteration we do not have to optimize the objective function, but an approximation to this function, such that the resulting optimizer are simple to compute. Let us therefore introduce next the approximation we intend to apply to our objective function and also derive with a theorem an analytic expression for the solution that maximizes it.

Suppose that $\mathbf{p}$ is "close" to some nominal parameter vector $\tilde{\mathbf{p}}$ and write $\mathbf{p}=\tilde{\mathbf{p}}+\Delta \mathbf{p}$, where $\Delta \mathbf{p}$ denotes a vector of perturbation. Suppose also that the intensity function $I_{w}$ and the warping transformation $T$ are of sufficient smoothness to allow for the existence of the required partial derivatives. If we denote as $\tilde{\mathbf{x}}^{\prime}=T(\mathbf{x} ; \tilde{\mathbf{p}})$ the warped coordinates under the nominal parameter vector and $\mathbf{x}^{\prime}=T(\mathbf{x} ; \mathbf{p})$ under the perturbed, then, applying a first order Taylor expansion with respect to the parameters, we can write

$$
I_{w}\left(\mathbf{x}^{\prime}\right) \approx I_{w}\left(\tilde{\mathbf{x}}^{\prime}\right)+\left[\nabla_{\mathbf{x}^{\prime}} I_{w}\left(\tilde{\mathbf{x}}^{\prime}\right)\right]^{t} \frac{\partial T(\mathbf{x} ; \tilde{\mathbf{p}})}{\partial \mathbf{p}} \Delta \mathbf{p}
$$

where $\nabla_{\mathbf{x}^{\prime}} I_{w}\left(\tilde{\mathbf{x}}^{\prime}\right)$ denotes the gradient vector of length 2 of the intensity function $I_{w}\left(\mathbf{x}^{\prime}\right)$ of the warped image evaluated at the nominal coordinates $\tilde{\mathbf{x}}^{\prime}$, and $\frac{\partial T(\mathbf{x} ; \tilde{\mathbf{p}})}{\partial \mathbf{p}}$ denotes the size $2 \times N$ Jacobian matrix of the warp transform with respect to its parameters, evaluated at the nominal values $\tilde{\mathbf{p}}$.

By applying (9) to all points of target area $\mathcal{S}$, forming the linearized version of the warp vector $\mathbf{i}_{w}(\mathbf{p})$ and computing its zero mean counterpart we obtain the following approximation $\rho(\Delta \mathbf{p} \mid \tilde{\mathbf{p}})$ of the objective function $\rho(\mathbf{p})$ defined in (8):

$$
\rho(\mathbf{p}) \approx \rho(\Delta \mathbf{p} \mid \tilde{\mathbf{p}})=\frac{\hat{\mathbf{i}}_{r}^{t} \overline{\mathbf{i}}_{w}+\hat{\mathbf{i}}_{r}^{t} \bar{G} \Delta \mathbf{p}}{\sqrt{\left\|\overline{\mathbf{i}}_{w}\right\|^{2}+2 \overline{\mathbf{i}}_{w}^{t} \bar{G} \Delta \mathbf{p}+\Delta \mathbf{p}^{t} \bar{G}^{t} \bar{G} \Delta \mathbf{p}}}
$$

where $G(\tilde{\mathbf{p}})$ denotes the size $K \times N$ Jacobian matrix of the warped intensity vector with respect to the parameters, evaluated at the nominal parameter values $\tilde{\mathbf{p}}$, and $\bar{G}$ denotes its column-zero-mean counterpart. Notice that for notational simplicity, the dependence of the warped vectors on $\mathbf{p}$ has been dropped. 
Although $\rho(\Delta \mathbf{p} \mid \tilde{\mathbf{p}})$ is a non-linear function of $\Delta \mathbf{p}$, its maximization results in a closed-form solution. This solution is given, without proof, by the next theorem.

Theorem I: Consider the scalar function

$$
f(\mathbf{x})=\frac{u+\mathbf{u}^{t} \mathbf{x}}{\sqrt{v+2 \mathbf{v}^{t} \mathbf{x}+\mathbf{x}^{t} Q \mathbf{x}}}
$$

where $u, v$ are scalars; $\mathbf{u}, \mathbf{v}$ are vectors of length $N ; Q$ is a square, symmetric and positive definite matrix of size $N$ and $v, \mathbf{v}, Q$ are such that

$$
v>\mathbf{v}^{t} Q^{-1} \mathbf{v}
$$

then, as far as the maximal value of $f(\mathbf{x})$ is concerned, we distinguish the following two cases:

Case $u>\mathbf{u}^{t} Q^{-1} \mathbf{v}$ : here we have a maximum, specifically

$$
\max _{\mathbf{x}} f(\mathbf{x})=\sqrt{\frac{\left(u-\mathbf{u}^{t} Q^{-1} \mathbf{v}\right)^{2}}{v-\mathbf{v}^{t} Q^{-1} \mathbf{v}}+\mathbf{u}^{t} Q^{-1} \mathbf{u}},
$$

which is attainable for

$$
\mathbf{x}=Q^{-1}\left\{\frac{v-\mathbf{v}^{t} Q^{-1} \mathbf{v}}{u-\mathbf{u}^{t} Q^{-1} \mathbf{v}} \mathbf{u}-\mathbf{v}\right\} .
$$

Case $u \leq \mathbf{u}^{t} Q^{-1} \mathbf{v}$ : here we have a supremum, specifically

$$
\sup _{\mathbf{x}} f(\mathbf{x})=\sqrt{\mathbf{u}^{t} Q^{-1} \mathbf{u}}
$$

which can be approached arbitrarily close by selecting

$$
\mathbf{x}=Q^{-1}\{\lambda \mathbf{u}-\mathbf{v}\}
$$

with $\lambda$ positive scalar, of sufficiently large value.

Using the results of Theorem I and by defining the projection matrix $P_{G}=\bar{G}\left(\bar{G}^{t} \bar{G}\right)^{-1} \bar{G}^{t}$, we have that the optimum perturbation is equal to

$$
\Delta \mathbf{p}^{o}=\left(\bar{G}^{t} \bar{G}\right)^{-1} \bar{G}^{t}\left\{\frac{\left\|\overline{\mathbf{i}}_{w}\right\|^{2}-\overline{\mathbf{i}}_{w}^{t} P_{G} \overline{\mathbf{i}}_{w}}{\hat{\mathbf{i}}_{r}^{t} \overline{\mathbf{i}}_{w}-\hat{\mathbf{i}}_{r}^{t} P_{G} \overline{\mathbf{i}}_{w}}-\overline{\mathbf{i}}_{w}\right\},
$$

when $\hat{\mathbf{i}}_{r}^{t} \overline{\mathbf{i}}_{w}>\hat{\mathbf{i}}_{r}^{t} P_{G} \overline{\mathbf{i}}_{w}$; or according to (16),

$$
\Delta \mathbf{p}^{o}=\left(\bar{G}^{t} \bar{G}\right)^{-1} \bar{G}^{t}\left\{\lambda \hat{\mathbf{i}}_{r}-\overline{\mathbf{i}}_{w}\right\},
$$

when $\hat{\mathbf{i}}_{r}^{t} \overline{\mathbf{i}}_{w} \leq \hat{\mathbf{i}}_{r}^{t} P_{G} \overline{\mathbf{i}}_{w}$, where $\lambda$ must be selected so that the resulting $\rho\left(\Delta \mathbf{p}^{o} \mid \tilde{\mathbf{p}}\right)$ satisfies $\rho\left(\Delta \mathbf{p}^{o} \mid \tilde{\mathbf{p}}\right)>\rho(0 \mid \tilde{\mathbf{p}})$ and $\rho\left(\Delta \mathbf{p}^{o} \mid \tilde{\mathbf{p}}\right) \geq 0$. Possible values of $\lambda$ provide the following lemma.

Lemma I: Let $\hat{\mathbf{i}}_{r}^{t} \overline{\mathbf{i}}_{w} \leq \hat{\mathbf{i}}_{r}^{t} P_{G} \overline{\mathbf{i}}_{w}$ and define the following two values for $\lambda$

$$
\lambda_{1}=\sqrt{\frac{\overline{\hat{\mathbf{i}}_{w}^{t} P_{G} \overline{\mathbf{i}}_{w}}}{\hat{\mathbf{i}}_{r}^{t} P_{G} \hat{\mathbf{i}}_{r}}}, \quad \lambda_{2}=\frac{\hat{\mathbf{i}}_{r}^{t} P_{G} \overline{\mathbf{i}}_{w}-\hat{\mathbf{i}}_{r}^{t} \overline{\mathbf{i}}_{w}}{\hat{\mathbf{i}}_{r}^{t} P_{G} \hat{\mathbf{i}}_{r}} .
$$

Then for $\lambda \geq \lambda_{1}$ we have that $\rho\left(\Delta \mathbf{p}^{o} \mid \tilde{\mathbf{p}}\right)>\rho(0 \mid \tilde{\mathbf{p}})$; for $\lambda \geq \lambda_{2}$ that $\rho\left(\Delta \mathbf{p}^{o} \mid \tilde{\mathbf{p}}\right) \geq 0$; finally for $\lambda \geq \max \left\{\lambda_{1}, \lambda_{2}\right\}$ we have both inequalities valid.

Let us now translate the above results into an iterative scheme in order to obtain the solution to the original nonlinear optimization problem.

To this end, let us assume that from iteration $j-1$ we have available the parameter estimate $\mathbf{p}_{j-1}$ and we adopt the following additive rule

$$
\mathbf{p}_{j}=\mathbf{p}_{j-1}+\Delta \mathbf{p}_{j} .
$$

Then, using $\mathbf{p}_{j-1}$ we can compute $\overline{\mathbf{i}}_{w}\left(\mathbf{p}_{j-1}\right)$ and $\bar{G}\left(\mathbf{p}_{j-1}\right)$ and optimize the approximation $\rho\left(\Delta \mathbf{p}_{j} \mid \mathbf{p}_{j-1}\right)$ with respect to $\Delta \mathbf{p}_{j}$. The iterative algorithm is summarized below. Initialization

- Use $I_{r}$ to compute $\hat{i}_{r}$ defined in (3).

- Initialize $\mathbf{p}_{0}$ and set $j=1$.

Iteration Steps

- Using $T\left(\mathbf{x} ; \mathbf{p}_{j-1}\right)$ warp $I_{w}$ and compute $\overline{\mathbf{i}}_{w}\left(\mathbf{p}_{j-1}\right)$

- Using $T\left(\mathbf{x} ; \mathbf{p}_{j-1}\right)$ warp the gradient $\nabla I_{w}$ of $I_{w}$ and compute the Jacobian matrix $\bar{G}\left(\mathbf{p}_{j-1}\right)$

- Compare $\hat{\mathbf{i}}_{r}^{t} \overline{\mathbf{i}}_{w}$ with $\hat{\mathbf{i}}_{r}^{t} P_{G} \overline{\mathbf{i}}_{w}$ and compute perturbations $\Delta \mathbf{p}_{j}^{o}$ either from (17) or using (18) and (19)

- Update parameter vector $\mathbf{p}_{j}=\mathbf{p}_{j-1}+\Delta \mathbf{p}_{j}^{o}$.

If $\left\|\Delta \mathbf{p}_{j}^{o}\right\| \geq \epsilon_{p}$ then, $j++$ and repeat; else stop.

As it is indicated above, the algorithm is executed until the norm of the perturbation vector $\left\|\Delta \mathbf{p}_{j}^{o}\right\|$ becomes smaller than a predefined threshold $\epsilon_{p}$.

Although, the structure of the iterative algorithm is very similar to the forward additive scheme of the Lucas-Kanade (LK) algorithm [10], one of the most frequently used algorithm for the image alignment problem, as we are going to see in the next section, our updating scheme improves the performance significantly.

\section{Simulation Results}

In this section we are going to evaluate our algorithm and compared it against the forward additive version of the Lucas-Kanade algorithm [10], as it is implemented in [3]. In all the experiments for the modelling of the warping process the class of projective transformation is used while a nonlinear photometric transformation is used for image distortion. We must stress at this point that for all aspects affecting the simulation experiments, we made an effort to stay exactly within the frame specified in [3]. Before we present our results we give some details for the experimental setup as well as the figures of merit we are going to use. 


\subsection{Parametric and Photometric Distor- tion Models}

In all experiments, to model the warping process we are going to use the projective transformation (homography) defined as follows

$$
\mathbf{x}^{\prime}=T(\mathbf{x} ; \mathbf{p})=\frac{1}{P}\left[\begin{array}{lll}
p_{1} & p_{2} & p_{3} \\
p_{4} & p_{5} & p_{6}
\end{array}\right]\left[\begin{array}{c}
\mathbf{x} \\
1
\end{array}\right]
$$

where $P=p_{7} x+p_{8} y+1$. This class of transformations is the most general class of the well known 2-D planar motion models that subsumes the most commonly used class of the affine transformations. For the Jacobian of the projective model we have

$\frac{\partial T(\mathbf{x} ; \mathbf{p})}{\partial \mathbf{p}}=\frac{1}{P}\left[\begin{array}{cccccccc}x & y & 1 & 0 & 0 & 0 & -x^{\prime} x & -x^{\prime} y \\ 0 & 0 & 0 & x & y & 1 & -y^{\prime} x & -y^{\prime} y\end{array}\right]$,

where $x^{\prime}, y^{\prime}$ are the elements of $\mathbf{x}^{\prime}$. As it is obvious from (22), projective model has a Jacobian that depends on the parameter $\mathbf{p}$, and thus it must be updated on each iteration of the iterative algorithm.

In order to investigate the performance of the two algorithms under photometrically distorted images, we consider nonlinear transformations of the following form

$$
I_{r}(\mathbf{x}) \leftarrow\left(I_{r}(\mathbf{x})+\alpha_{2}\right)^{\gamma}, \mathbf{x} \in \mathcal{T}
$$

which are applied to the intensity of each pixel in the reference image. In the experiments presented in this work we used $\alpha_{2}=10$ and $\gamma=0.9$ respectively. Notice that in order not to favor the competing algorithms which are invariant to linear photometric distortions, we adopt a nonlinear photometric distortion model.

\subsection{Experimental Setup}

The experimental setup is described analytically in [3]. In brief, we have an input image $I_{0}$ and we crop a rectangular area of the image. By adding an appropriate translation in the coordinates of the points corresponding to the corners of the cropped image and adding Gaussian noise with standard deviation $\sigma_{p}$ we perturb them. The four initial points, and their warped versions defines the parameter vector of the projective transformation. Using these values, we map all target points and warp $I_{0}$ to define a reference image $I_{r}$. The competing algorithms then are applied for the alignment of $I_{r}$ with $I_{0}$.

In order to measure the quality of the estimated parameters we use the mean square distance (MSD) between the exact warped version of the four points and their estimated counterparts. More formally, if we denote as $\mathbf{p}_{r}$ the parameter vector which we use in defining the reference profile $I_{r}, \mathbf{p}_{j}$ the current estimation of the corresponding algorithm at $j$-th iteration and $\mathcal{C}$ the set of the four above mentioned points, we use the mean of the following sequence

$$
\bar{e}(j)=\frac{1}{8} \sum_{\mathbf{x} \in \mathcal{C}}\left\|T\left(\mathbf{x} ; \mathbf{p}_{r}\right)-T\left(\mathbf{x} ; \mathbf{p}_{j}\right)\right\|^{2} .
$$

Each element of the mean sequence (i.e. for a specific value of iteration index $j$ ) is obtained by averaging over a large number of image pairs that differ in the noise realization, and captures the learning ability of the algorithms (average rate of convergence [3]). However, in order to not present biased results, we compute the above mentioned mean sequence for those realizations where both algorithms have converged. The convergence criterion is that the MSD at a prescribed maximal iteration $j_{\max }$ is below a certain threshold $T_{M S D}$, that is $\bar{e}\left(j_{\max }\right) \leq T_{M S D}$.

Since it is natural to prefer an algorithm that converges quickly with high probability, we propose a second figure of merit that captures exactly this point. In other words we propose the generation of a histogram depicting the probability of successful convergence at each iteration. Specifically a run of an algorithm on an image pair realization will be considered as having converged at iteration $n$ when the squared error $\bar{e}(j)$ goes below the threshold $T_{M S D}$ for the first time at iteration $j=n$. It is clear that we prefer a histogram to be concentrated (or equivalently the probability of successful convergence to be distributed) over mostly small iteration-numbers. In all experiments that follow we use $T_{M S D}=1$ pixel $^{2}$.

Finally, a third figure of merit we use is the percentage of converging (PoC) runs (frequency of convergence [3]). This quantity is the percentage of runs that converge up to maximal iteration $j_{\max }$, based again on the above convergence criterion. PoC is depicted as a function of the point deviation $\sigma_{p}$, the most important factor that affects the performance of both algorithms.

\subsubsection{Experiment I}

In the first experiment, the alignment algorithms try to compensate both the geometric and photometric distortions that have been applied to images. Specifically, we use the "Takeo" image [3] as the warped profile image and by using the procedure described in Subsection 4.2 we generate a reference image which in the sequel is photometrically distorted as described in (23). We make 500 realizations of image pairs and run both the algorithms for all integer values of $\sigma_{p}$ in the range $[1,10]$, and compare their convergence characteristics for a maximum number of iterations $j_{\max }=15$.

Figure 1 depicts the relative performance of the two algorithms. In the computation of all figures of merit we have taken into account the realizations where both algorithms have converged. Three cases are investigated; (a) $\sigma_{p}=2$, 
(b) $\sigma_{p}=6$ and (c) $\sigma_{p}=10$. In all cases the proposed algorithm exhibits a significantly smaller MSD which is order(s) of magnitude better than the one obtained by using the LK scheme. Furthermore concerning the PoC, as we can see from Figure 1.(d), our algorithm exhibits better performance for all values of $\sigma_{p}$. Specifically for medium and strong geometric deformations $\left(\sigma_{p}=6\right.$ and $\sigma_{p}=10$, respectively) the improvement can become quite significant ( $10 \%$ and $18 \%$ respectively). Regarding the second figure
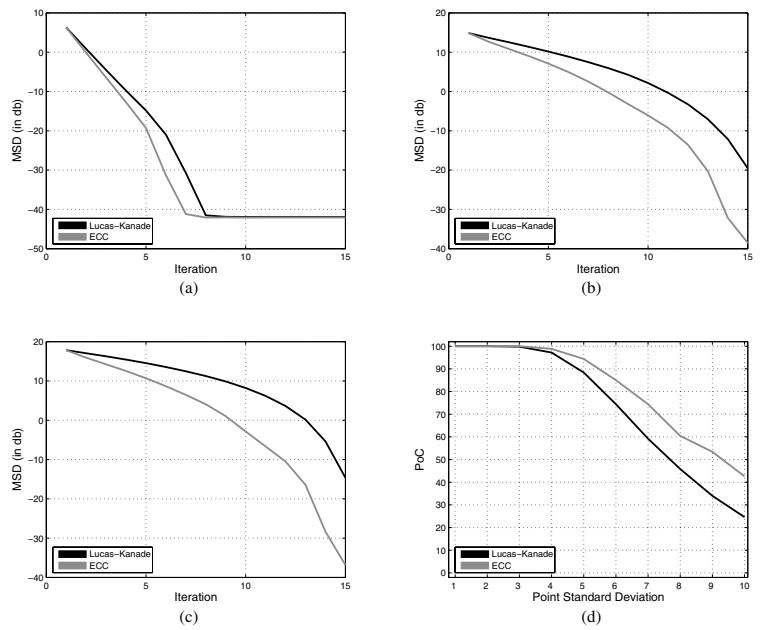

Figure 1. MSD in $\mathrm{dB}$ as a function of number of iterations; (a) $\sigma_{p}=2$, (b) $\sigma_{p}=6$, (c) $\sigma_{p}=10$. In (d), PoC as a function of $\sigma_{p}$ for $j_{\max }=15$.

of merit, that is the histogram of successful convergence, we applied the algorithms for a maximal number of iterations $j_{\max }=100$. In Figure 2 the resulting graphs are shown. In order however, for the differences to become visible, we present only the first 50 bins of the histogram. The left graph of Figure 2 has the histogram for the case of $\sigma_{p}=6$ and the right for $\sigma_{p}=10$. We can clearly see that our algorithm has larger percentage of converged realizations in smaller iteration numbers than the LK scheme.

\subsubsection{Experiment II}

In this experiment, we repeat the simulation of the previous case, but now we add intensity noise to both images before their alignment. Specifically, the standard deviation of the noise we add into the images is equal to 8 gray levels.

In Figure 3 the simulation results we obtained are shown. For the case of $\sigma_{p}=2$ we observe that the algorithms reach a different MSD floor value, with the proposed algorithm achieving a floor value which is more than $3-d B$ lower than the floor value achieved by the LK algorithm. Notice also that in all cases our algorithm outperforms the LK scheme
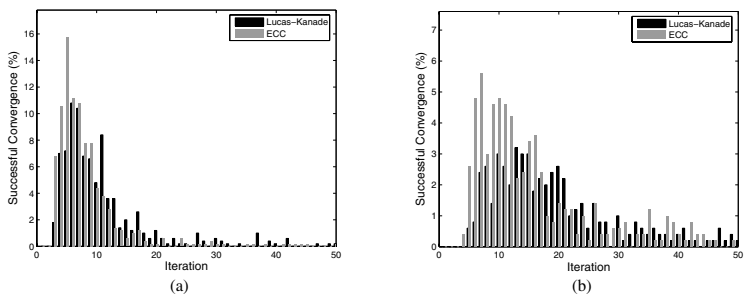

Figure 2. Intensity noise-free case. Histogram of successful convergence as a function of iteration number; left graph correspond to $\sigma_{p}=6$ and right to $\sigma_{p}=10$ for the photometrically distorted Takeo image.

by a half or a full order of magnitude. Furthermore, ECC algorithm exhibits a larger $\mathrm{PoC}$ score confirming thus its superiority.
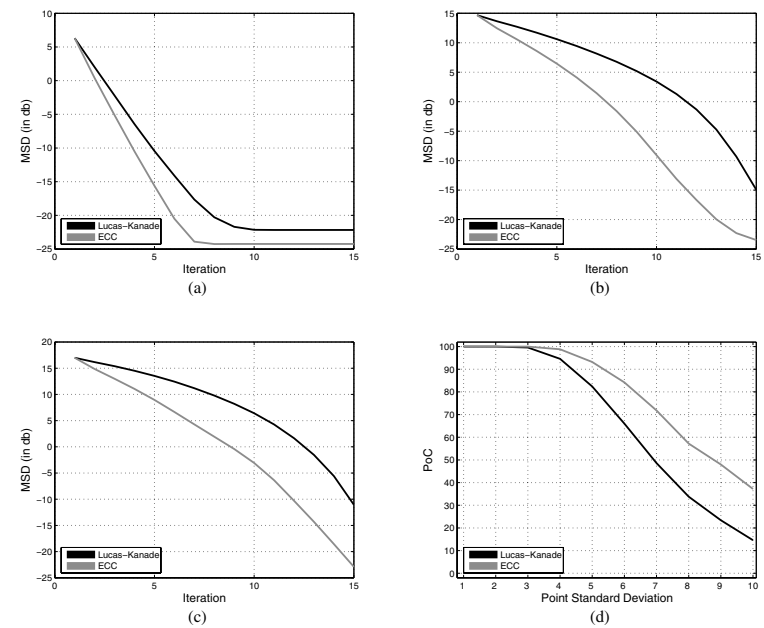

Figure 3. MSD in $\mathrm{dB}$ as a function of number of iterations for the noisy (8 gray lelels) "Takeo" image; (a) $\sigma_{p}=2$, (b) $\sigma_{p}=6$, (c) $\sigma_{p}=10$. In (d), PoC as a function of $\sigma_{p}$ for $j_{\text {max }}=15$.

Similar conclusion can be drawn from Figure 4 that depicts the two histograms. We can see that the histogram of our algorithm is very similar to the previous intensity noise-free case and concentrated at smaller iteration numbers while the LK is almost uniformly spread over the range 5 to 50 .

Concluding, from a large number of experiments we have conducted, we can safely say that the proposed iterative scheme outperforms the LK algorithm in all three figures of merit, also exhibiting a noticeable robustness to inaccurate nonlinear photometric distortion modelling. This latter characteristic is not enjoyed by the LK iterative 

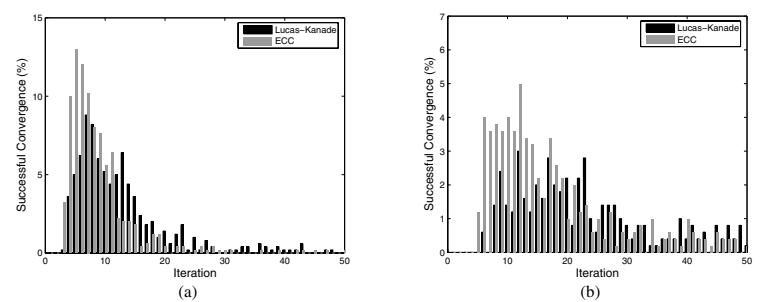

Figure 4. Intensity noise case. Histogram of successful convergence as a function of iteration number; left graph correspond to $\sigma_{p}=6$ and right to $\sigma_{p}=10$ for the photometrically distorted Takeo image.

scheme.

\section{Conclusions}

In this work a recently proposed alignment algorithm was used in the projective registration problem. This algorithm aims at maximizing the Enhanced Correlation Coefficient function which is a robust similarity measure against both geometric and photometric distortions. The optimal parameters are obtained by solving, iteratively, a sequence of approximate nonlinear optimization problems, which enjoy a simple closed-form solution with low computational cost. Regarding the applicability of the algorithm, two interesting experiments with photometrically distorted images were investigated. The iterative algorithm was compared against the Lucas-Kanade algorithm, through numerous simulation examples involving ideal and noisy conditions and strong or weak geometric deformations. In all cases the proposed algorithm exhibited a better behavior with an improvement in speed, as well as in probability of convergence as compared to the Lucas-Kanade scheme.

\section{References}

[1] Y. Altunbasak, R. M. Mersereau, and A. J. Patti. A fast parametric motion estimation algorithm with illumination and lens distortion correction. IEEE Transactions on Image Processing, 12(4):395-408, 2003.

[2] P. Anandan. A computational framework and an algorithm for the measurement of visual motion. International Journal of Computer Vision, 2(3):283-310, 1989.

[3] S. Baker and I. Matthews. Lucas-kanade 20 years on: A unifying framework: Part 1: The quantity approximated, the warp update rule, and the gradient descent approximation. International Journal on Computer Vision, 56(3):221-255, 2004.

[4] M. J. Black and P. Anandan. A framework for the robust estimation of optical flow. In Proc. of 4th IEEE International
Conference on Computer Vision (ICCV'93), 1993. Berlin, Germany.

[5] G. D. Evangelidis and E. Z. Psarakis. Parametric image alignment using enhanced correlation coeeficient maximization. submitted to IEEE Trans. on PAMI, submission TPAMI0026-0107.

[6] C. Fuh and P. Maragos. Motion dislpacement estimation using an affine model for image matching. Optical Engineering, 30(7):881-887, 1991.

[7] G. D. Hager and P. N. Belhumeur. Efficient region tracking with parametric models of geometry and illumination. IEEE Trans. on PAMI, 20(10):1025-1039, 1998.

[8] B. K. P. Horn and B. G. Schunk. Determining optical flow. Artificial Intelligence, 17:185-203, 1981.

[9] B. K. P. Horn and E. J. Weldon. Direct methods for recovering motion. International Journal of Computer Vision, 2:5176, 1988.

[10] B. D. Lucas and T. Kanade. An iterative image registration technique with an application to stereo vision. In Proc. of 7th International Joint Conf on Artificial Intelligence (IJCAI), 1981. Vancouver, British Columbia.

[11] E. Z. Psarakis and G. D. Evangelidis. An enhanced correlation-based method for stereo correspondence with sub-pixel accuracy. In Proc. of 10th IEEE International Conference on Computer Vision (ICCV 2005), 2005. Beijing, China.

[12] H. Shum and R. Szeliski. Construction of panoramic image mosaics with global and local alignment. International Journal on Computer Vision, 36(2):101-130, 2000.

[13] R. Szeliski. Handbook of Mathematical Models of Computer Vision. Springer, 2005. ch. 17. 